2.

\title{
Sanitäre Gedanken über den Chiton der Homerischen Helden.
}

\author{
Von Oberstabsarzt H. Frölich in Dresden.
}

So jugendlich sich auch die Gesundheitskunde in der Reihe der medicinischen Wissenschaftsgebiete heutzutage ausnimmt, so weit zuröck lässt sich doch ihre Anwendung auf's Leben verfolgen. Die Aussenseite des Alltagslebens gilt eben schon jenen culturarmen Perioden des Alterthums keineswegs als ein Paradies, in welchem sich Jeder nach Gefallen anfechtungslos umbertummeln könne, sondern als eine terra aspera, aus welcher beständig eine üppige Saat von Gefahren für Gesundheit und Leben aufgehe.

Im Sinne dieser Anschauung umgab man sich gegen diese Gefahren mit Schutzwehren und verlegte die letzteren - zumal gegenüber den Kriegsfäbrlichkeiten zum guten Theil in die nächste Ungebung des Menschen, in die Kleidung. Die Kleidung hatte also bei den ältesten Völkern und insbesondere bei den ím Folgenden in Betracht kommenden Griechen, den Hauptzweck, für das menschliche Individuum gegen natürliche (namentlich klimatische) und künstliche Angriffe eine Wehr zu bilden; und sie verschwamm mit der Rüstung fast in ein zusammenbängendes Ganze, wenn eine solche Angriffsgefahr rom bewaffneten Feinde her drohte.

Dabel war die zweckdienliche Bekleidung der Griechen böchst einfach; jede überflüssige Zuthat hätte den griechischen Schönheitssinn verletzt, welcher sich gegen vollständige Umhüllung sträubte und das Ebenmaass der Glieder dem Auge nicht verloren gehen lassen wollte. Dass aber, wie man neuerdings behauptet hat, die Bekleidung vermöge des Klimas überhaupt nur insoweit existirt hätte, als es das Anstandsgefühl erforderte, ist eine so ästhetische wie unsanitäre Ansicbt, welche durch die Lectüre der literarischen Ueberlieferungen nicht unterstützt werden kann. Man hatte vielmehr, wie auch aus der folgenden Darstellung nebenbei hervorgebt, ganze Gewänder, welehe einen Kunstfleiss voraussetzen, der spottend über adamitischer Kindlichkeit steht.

Den Grad dieses Kunstfleisses labe ich für die Kopfbedeckung der Homerischen Helden in diesem Archive (Jahrg. 1876, 68. Bd., 3. Heft) festzustellen versucht; im Nachstehenden soll ein zielgleicher Nachweis für das allgemeinste Bekleidungsstūck der Altgriechen, für den $\chi \iota t \omega \nu$, vom sanitären Standpunkte aus gefübrt werden.

Was war der $\chi \iota \tau \omega \nu$ ? Voss übersetzt dieses Wort mit „Leibrock“, aber auch mit "Panzer"! In ähnlicher Weise geben die neueren Wörterbücher das Wort wieder und fügen mit anscheinend besserer Einsicht den Ausdruck "Unterkleid " hinzu. Es ist also der Begriff $\chi \iota \tau \omega \nu$ entweder mehrsinnig gebraucht worden, oder es fehlt uns heute an dem richtigen Verständnisse oder nur an einer deckenden Bezeichnung.

Diesem Sachverhalte gegenüber ist es für die Gesundheitslehre, welche ja immer mit mehr oder weniger Nutzen die der Gesunderhaltung dienenden Maass- 
regeln anderer Zeiten und Völker mit den jetzigen und heimischen vergleichen wird, von Belang, wenn irgend möglich, das Dunkel aufzuhellen, welches den $\chi \bullet \tau \omega \nu$ des Homerischen Zeitalters theilweis ungiebt.

Das hierzu nöthige Licht spenden die Homer'schen Gesänge, welche, soweit ich mich erinoere, dem $\chi \iota t a v$ folgende Kennzeichen zuschreiben.

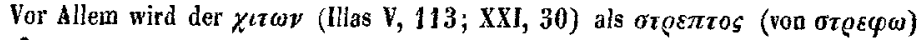
"gedreht" bezeichnet, so dass Voss das Ganze als "geflochtenen Panzer" auffast. Vorläufig lässt sich weder für noch gegen diese Uebersetzungsweise etwas aufbringen; nur ist die Möglichkeit, welche ich fïr meine abweichende Meinung vorweg andeuten möchte, nicht ausgeschlossen: dass otgentos bier im Sinne von, ,gewebt“ gebraucht sein und man somit im $\chi$. $\sigma \tau \varrho$. ein gewebtes Kleid vor sich haben kann.

Der Stand der damaligen Kunstfertigkeiten hinderte wenigstens nicht am Besilze gewebter Kleidungstücke. Das Spinnen und Weben verstand die Homerische Zeit: man kannte nicht nur die Spule $(\varkappa \alpha \nu \omega \nu)$ und Spindel ( $\left.\eta^{\prime} \lambda \alpha \alpha \tau \eta\right)$, sondern auch den Webstuhl (iбros), an welchem die Kette zum Weben senkrecht aufgezogen wurde, so dass die Fäden herabhingen und man beim Weben um den Stuhl herumgehen musste ${ }^{1}$ ). Die Männer des Krieges freilich lagen dieser Arbeit nicht ob; Hausfrauen und Mägde, aber auch Göttinnen (Il. XIV, 178) trieben dieses Stubenhandwerk.

Wie so vieles, enthält auch dieses Gebahren der Alten warnende Fingerzeige für das moderne Leben, indem es die sanitären Fragen veranlasst: ist es dem sanften Einflusse einer weisen Nationalökonomie nicht möglich, das Weben in der Stube wieder vorwiegend zur weiblichen Nebenbeschäftigung zn entwickeln? oder sollte es nicht wenigstens der Technik gelingen, das Weben wieder auf eine gebende Körperbaltung anzuweisen?

Einer weiteren, entscheidenden Eigenschaft des $\chi \iota z \omega \nu$ begegnet man In

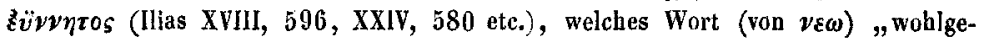
sponnen" bedeutet und die vorhin wider Voss ausgesprochene Möglichkeit zur Gewlssheit erhebt.

Auch das häufig (z. B. in Ilias II, 42;0d. 1, 437) vorkommende Beiwort $\mu \alpha \lambda \alpha x o$, ,weich", verbietet es im $\chi \iota t \omega \nu$ einen Panzer, also das, was Homer

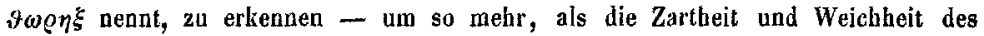
$\chi \iota 7 \omega \nu$ in 0 d. XIX, 233 mit der Schale einer getrockneten Zwiebel verglichen wird.

In scheinbarem Widerspruche mit diesen bisher erwähnten Eigenschaften steht

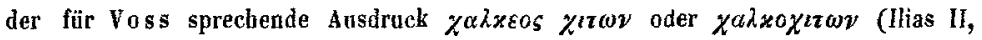
163 ; IV, 199; XIII, 440 etc.) - wörtlich: ein metallischer $\chi \imath \tau \omega \nu$. Allein es lässt sich auch dieser Begriff unschwer mit dem planzlichen Herkommen des $\chi^{\iota \tau \omega} \omega \nu$ vereinigen, wenn man sich nicht der Vorstellung hingiebt, dass ein metallischer $\chi \imath \tau \omega \nu$ ganz und gar aus Metall (Bronze) bestanden haben müsse. Wie ich dies schon für den Homerischen Helm nachgewiesen habe, wird der $\chi \imath \tau \omega \nu$ vorübergehend für kriegerische Vertheidigungszwecke, wahrscheinlich mit Metall, mit Metall-

7) Näheres hierüber wird sich in der ,Technologie und Terminologie der Gewerbe und Künste bel Griechen und Römern, H ug o Blümper in Breslau, Leipzig $187 \mathbf{j}^{\prime \prime}$ finden. 
platten ausgestattet worden sein, deren Befestigungsweise die Abnehmbarkeit derselben ermöglicht hat. Wenn diese Art der Ausrüstung eine den Rumpf umfassende Ausdehnung batte, so bat man gewiss das vor sich, was Homer (Ilias II, 529, 830 etc.) $\lambda \iota v 0 \vartheta \omega \emptyset \eta \xi$ pennt - einen Panzer mit leinener Auskleidung, zum Unterschiede vom einfachen $\vartheta \omega \varrho \eta \xi$, den man sich als ein selbstăndiges und durchaus ehernes Ausrüstungsstück vorstellen darf.

Wenn ich es als höchst wahrscheinlich betrachte, dass der $\chi \cdot \chi \alpha i x \varepsilon o s$ ein mit Metallstücken verstärkter $\chi \imath \tau \omega \nu$ gewesen 1st, so könnten mir es die in vorliegender Frage maassgeblicheren Sprachforscher doch nicht nachsehen, wenn ich daräber schwiege, wie ich mir die Befestigungsart dieser Metallplättchen denke. Stellt man Erwägungen hierüber an, so verfällt man unmittelbar auf das gebräuchlichste Binde- und Befestigungsmittel jener Zeit, auf den (Leder-) Riemen. Gewiss fand der Riemen deshalb eine so ausgedehnte Anwendung, weil er so sehr leicht berzustellen ist - was uns die Beschäftigung der neulich in Deutschland besuchsweise anwesenden Eskimos vor Augen geführt bat. Die blosse Abtrennung des schmalen Saumes eines Felles mit dem Messer genügt, in der kürzesten Zeit sich éinen Riemen zu verschaffen; und dieser Umstand, der gerade im Felde sehr in Anschlag zu bringen ist, leitet mich mit auf die Annahme, dass jene (vielleicht mit Oesen versehene) Metallplatten mittelst Riemen an den $\chi \iota \tau \omega \nu$ geknötet worden sind.

Gegen diese Auffassung könnte man den kräftigen Einwand erheben, dass Homer einer solchen Befestigangsart nirgends Erwähnang thut. Dagegen muss ich einhalten, dass allerdings eine bierauf bezügliche Stelle (llias XXI, 31) vorliegt, die bisher vielleicht nur unrichtig gedeutet worden ist.

Es wird nehmlich ebenda erzählt, wie Achilleus 12 trojanische Jünglinge gefangen nimmt und denselben die Hände auf den Rücken bindet mit Riemen, die sie an ihrem gewebten $\chi \imath \tau \omega \nu$ hatten. Aus dieser Stelle hat man verallgemeinernd den Schluss gezogen, dass die Krieger sich mit einem Vorrath von Riemen trugen zu dem Zwecke, Gefangene zu fesseln oder wie Andere wollen, zu beliebigem Gebrauche. Gegen beide Zwecke aber spricht aus dieser Stelle die Unterkunft dieser Biemen ,am gewebten $\chi \imath \tau \omega \nu^{66}$. An einem hinter der Ausrüstung unmittelbar dem Körper aufliegenden, also sehr schwer zugängigen Bekleidungsstücke wird man doch offenbar nicht Dinge aufgespeichert haben, welche man damals gewiss ebenso häufig gebrauchte, wie wir heutigen Tages etwa die Knöpfe. Ich kann mich deshalb nicht von der Auffassung trennen, dass jene Riemen am "gewebten $\chi \imath \tau \omega \nu^{\text {" }}$ nur den Zweck batten, metallische Verstärkungen am $\chi \imath t \omega \nu$ aufzuhängen. Ob man wirklich, an einer leichter zugängigen Stelle, noch einen Riemenvorrath mit sich getragen bat, lasse ich dahingestellt - möglich ist es, aber unbewiesen.

Jedenfalls hat der Riemen auch im Felde eine so allgemeine Nutzanwendung erfahren, dass er unwillkürlich zur Vergleichung mit seinen heutigen Ersatzmittela auffordert. Wir bedienen uns an seiner Stelle, auch im Feldsanitätsdienste, der Stricke, Bänder und Binden; - ob allenthalben mit mehr Recht? Das beantwortet die Thatsache, dass der Riemen mindestens ebenso fest und haltbar ist wie jene Ersatzmittel und dass er viel leichter zu beschaffen ist, als jene. Die fäulnigswidrig zuzubereitenden Hänte gefallener Pferde und geschlachteter Rinder liefern im Felde ein überreiches Riemenmaterial! 
Welchen Umfang der $\chi \iota \tau \omega \nu$ gehabt bat und wie viel er vom Körper bedeckt hat, das geht in erschöpfender Weise aus den Schilderungen Homer's nicht hervor. Hauptsächlich hat der $\chi ı x \omega \nu$ die Brust (z. B. Ilias II, 416; X, 21, 131) umspannt, ferner hat er unter den Brustharnisch (Ilias III, 359) herabgereicht; ja er muss mindestens noch einen Theil der Oberschenkel verhüllt haben, denn zur Arbeit und zum Kampfe gürtete man sich (llias XI, 15; XXIII, 685; 0d, XIV, 72) über den Hüften, was nur dann einen Zweck haben konnte, wenn der $\chi \iota t(x)$ noch zum guten Theile unterhalb des Gurtes hervorragte.

Der Gurt für den $\chi^{\imath \tau \omega \nu}$ ist wahrscheinlich (llias $1 V, 213-216$ ) die $\mu \iota \tau$ Q Homer's gewesen. Zwar ist dieselbe nach dieser Stelle von Erzarbeitern gefertigt, allein diese Angabe beziebt sich vermuthlich auf die metallische Verstärkung der $\mu \iota \tau \rho \alpha$, deren sie aus denselben Gründen bedurfte, wie der $\chi \iota \tau \omega \nu$.

Vor dem Schlafengehen löste man den Gurt und zog sich den $\chi \imath t \omega \nu$ aus, um sích mit schlechteren Wärmeleitern (Fellen etc., 0 d. 1, 443 etc.) zu bedecken. Vor dem Feinde freilich, im Biwak, wird man sich vor der gằnzlichen Entkleidung (0d. XIV, 480) für die Nacht wohl gehütet haben. -

Fasst man das über den $\chi t \tau \omega y$ Gesagte zusammen und zieht von Homer häufig gedachter Nebenumstände (z. B. die Reihenfolge des Ablegens und Anlegens der Kleidungsstïcke etc.) mit in Betracht, so gelangt man zu der Ueberzeugung, dass der $\chi \imath t \omega \nu$ an sich weder ein Rock noch ein Panzer, sondern ein "Hemd" gewesen ist, welches man für kriegerische Vertheidigungszwecke mit metallischen (bronzenen) Verstärlsungsmitteln auszustatten und so in ein "Panzerhemd“ ( $x_{2}$ \% $\chi \alpha \lambda x \varepsilon \circ \varsigma, \lambda(\nu \circ \vartheta \omega \varrho \eta \xi)$ umzuwandeln verstanden hat. Näberes über seine Gestalt ist den Homerischen Gesängen nicht zu entlehnen. Wahrscheinlich hat er, wie Guhl und Koner, gestützt auf andere spätere Quellen, meinen, aus einem Stück Zeuge bestanden, welches einfach zusammengelegt an dem blinden Ende des oberen Randes ein Aermelloch für die Aufnahme des (linken) Armes hatte, während die gegenüberliegenden Enden die entgegengesetzte Schulter umspanuten und bier durch eine Spange vereinigt wurden.

Was endlich den eigentlichen Zweck des $\chi \imath \tau \omega \nu$ anlangt, so kann man aus dem, vornehmlich auf die Bedeckung des Rumpfes abzielenden beträchtlichen Umfange dieses Hemdes nicht ableiten, dass seine Erfindung lediglich dem Anstandsgefühle zu verdanken sei. Viejmehr weist das Tragen des $\chi i z c u v$ im Freien und die nächtliche Vertanschung desselben mit einem schlechteren Wärmeleiter darauf hin, dass ihn der Zweckmässigkeitssinn der Griechen rechtfertigte - derselbe Sinn, welcher mit der heutigen pbysikalischen Anschaung von der Steigerung der Wärmeleitung bei Bewegung im Freien and von der Verminderung der körperlichen Wärmeerzeugung beim Schlafen zusammenfallen mag.

Eine sachverständige Uebersetzung der, mit reizvoller Naturwahrheit die Wirklichkeit des Lebens schildernden Gesänge Homer's ist somit zugleich eine lehrreiche Uebersetzung der gesundheitlichen Erfahrungssätze jener Zeit in die experimentell erhärteten Lehrsätze der beutigen Gesundheitspflege! 